\title{
Draft
}

\section{Spanning Trees with Leaf Distance at Least Four}

\author{
Atsushi Kaneko, M. Kano ${ }^{1}$ and Kazuhiro Suzuki ${ }^{1}$ \\ ${ }^{1}$ Department of Computer and Information Sciences \\ Ibaraki University \\ Hitachi, Ibaraki 316-8511 Japan \\ e-mail: kano@cis.ibaraki.ac.jp
}

\begin{abstract}
For a graph $G$, we denote by $i(G)$ the number of isolated vertices of $G$. We prove that for a connected graph $G$ of order at least five, if $i(G-S)<|S|$ for all $\emptyset \neq S \subseteq V(G)$, then $G$ has a spanning tree $T$ such that the distance in $T$ between any two leaves of $T$ is at least four. This result was conjectured by Kaneko in "Spanning trees with constrains on the leaf degree", Discrete Applied Math., 115 (2001) 73-76. Moreover, the condition in the result is sharp in a sense that the condition $i(G-S)<|S|$ cannot be replaced by $i(G-S) \leq|S|$.
\end{abstract}

\section{Introduction}

We consider finite graphs which have neither multiple edges nor loops. Let $G$ be a graph with vertex set $V(G)$ and edge set $E(G)$. We denote by $I(G)$ the set of isolated vertices of $G$, and by $i(G)$ the number of isolated vertices of $G$, that is, $i(G)=|I(G)|$. The degree of a vertex $v$ in $G$ is denoted by $\operatorname{deg}_{G}(v)$. A vertex of degree one is called a pendant vertex, and a pendant vertex of a tree is usually called a leaf. An edge incident with an pendant vertex is called a pendant edge.

There are many results on the existence of spanning trees with some given properties. For example, Win [5] proved that if $\omega(G-S) \leq(k-2)|S|+2$ for all $\emptyset \neq S \subseteq V(G)$, then $G$ has a spanning tree with maximum degree at most $k$, where $k \geq 2$ and $\omega(G-S)$ denotes the number of components of $G-S$. Ellingham, Nam and Voss [3] showed that every $m$-edge connected graph has spanning tree $T$ such that $\operatorname{deg}_{T}(v) \leq 2+\left\lceil\operatorname{deg}_{G}(v) / m\right\rceil$ for every vertex $v$ of $G$. Other similar results can be found a recent survey [4] by Kouider and Vestergaard.

In this paper, we consider a spanning tree with given leaf distance. Let $T$ be a spanning tree of a graph. The leaf distance of $T$ is defined to be the minimum of distances between any two leaves of $T$ (see Figure 1). The leaf degree of a vertex $v$ in $T$ is the number of leaves of $T$ incident with $v$, and the maximum leaf degree of $T$ is the maximum leaf degree among the vertices of $T$. 
Kaneko made the following conjecture.

Conjecture 1 ([1]) Let $d \geq 4$ be an integer and $G$ be a connected graph with order at least $d+1$. If

$$
i(G-S)<\frac{2|S|}{d-2} \quad \text { for all } \quad \emptyset \neq S \subseteq V(G),
$$

then $G$ has a spanning tree with leaf distance at least $d$.

Moreover, he showed that if the above conjecture is true for an even integer $d$, then the condition (1) is sharp in a sense that there exist connected graphs $G^{\prime}$ that have no spanning tree with leaf distance at least $d$ and satisfy $i\left(G^{\prime}-S\right) \leq 2|S| /(d-2)$ for all $\emptyset \neq S \subseteq V\left(G^{\prime}\right)$. Other class of such graphs for $d=4$ is the following: the corona $\operatorname{cor}\left(K_{n}\right)$ of a complete graph $K_{n}$, which is obtained from $K_{n}$ by adding a pendant edge to each vertex of $K_{n}$, satisfies $i\left(\operatorname{cor}\left(K_{n}\right)-X\right) \leq|X|$ for every $\emptyset \neq X \subseteq V\left(\operatorname{cor}\left(K_{n}\right)\right)$, but has no spanning tree with leaf distance at least four.

Note that Conjecture 1 is true for $d=3$ by the following theorem with $m=1$ since the leaf distance of a spanning tree $T$ is at least three if and only if the maximum leaf degree of $T$ is one.

Theorem 2 ([1]) Let $G$ be a connected graph and $m \geq 1$ be an integer. Then $G$ has a spanning tree with maximum leaf degree at most $m$ if and only if

$$
i(G-S)<(m+1)|S| \quad \text { for all } \emptyset \neq S \subseteq V(G) .
$$

In this paper, we shall prove the following theorem, which implies that Conjecture 1 is true for $d=4$.

Theorem 3 Let $G$ be a connected graph with order at least five. If

$$
i(G-S)<|S| \quad \text { for all } \emptyset \neq S \subseteq V(G),
$$

then $G$ has a spanning tree with leaf distance at least four (Figure 1).

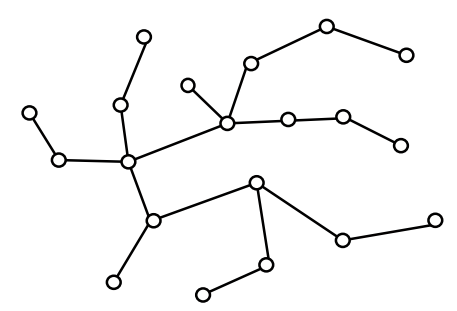

Figure 1: A tree with leaf distance four 


\section{Proof of Theorem 3}

Let $G$ be a graph. For a subset $S \subseteq V(G)$, we write $G-S$ for the subgraph of $G$ induced by $V(G)-S$. For two disjoint vertex subsets $S$ and $T$ of $G$, we denote by $E_{G}(S, T)$ the set of edges of $G$ joining a vertex in $S$ to a vertex in $T$. We denote by $N_{G}(v)$ the neighborhood of

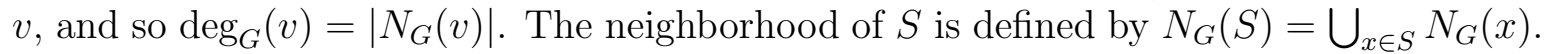
We call a component with order at least two a non-trivial component.

For a set $X$ and its subset $Y$, we write $Y \subset X$ when $Y$ is a proper subset of $X$.

Lemma 4 (Kaneko and Yoshimoto [2]) Let $G$ be a connected bipartite graph with bipartition $A \cup B$, and $f$ be a function defined by $f: A \rightarrow\{2,3,4, \ldots\}$. Then $G$ has a spanning tree $T$ such that $\operatorname{deg}_{T}(x) \geq f(x)$ for all $x \in A$ if and only if

$$
\left|N_{G}(S)\right| \geq \sum_{x \in S} f(x)-|S|+1 \quad \text { for all } \emptyset \neq S \subseteq A .
$$

In particular, if $\left|N_{G}(S)\right| \geq|S|+1$ for all $\emptyset \neq S \subseteq A$, then $G$ has a spanning tree all whose leaves are contained in $B$.

Proof of Theorem 3. We prove Theorem 3 by induction on the lexicographic order of $(|V(G)|,|E(G)|)$, and thus, when we consider a graph $G$, we may assume that the theorem holds for a graph $H$ with either $|V(H)|<|V(G)|$ or $|V(H)|=|V(G)|$ and $|E(H)|<|E(G)|$. By (3), $G$ has no pendant vertices, that is, $\operatorname{deg}_{G}(x) \geq 2$ for every $x \in V(G)$. We often use this fact without mentioning it.

Claim 1. We may assume that $G$ has order at least eight.

If $G$ has order 5 or 6 , then we can easily show that $G$ has a Hamiltonian path, which is obviously the desired spanning tree. Suppose that $G$ has order 7 . In this case, we shall show that $G$ has a Hamiltonian path, or $G$ is a graph with vertex set $\left\{u, v_{1}, v_{2}, v_{3}, v_{4}, v_{5}, v_{6}\right\}$ and edge set $\left\{u v_{i} \mid 1 \leq i \leq 6\right\} \cup\left\{v_{1} v_{2}, v_{3} v_{4}, v_{5} v_{6}\right\}$, which is called the 7-windmill and has a spanning tree with leaf distance four. Let $k$ be the length of a longest cycle of $G$. If $k \geq 4$, then by considering the cases of $k=7,6,5$ and $k=4$ one by one, we can show that $G$ has a Hamiltonian path. If $k=3$, then we can show that $G$ has a Hamiltonian path or $G$ is the 7 -windmill. Hence Claim 1 follows.

Since every vertex has degree at least two, we have $|E(G)| \geq|V(G)|$. If $|E(G)|=$ $|V(G)|$, then $G$ must be a cycle, and so $G$ has a Hamiltonian path, which is obviously the desired spanning tree. Hence we may assume $|E(G)| \geq|V(G)|+1$, in particular, $G$ has an edge $e_{0}$ such that $G-e_{0}$ is connected.

Claim 2. There exists a subset $\emptyset \neq R \subset V(G)$ such that $1 \leq|R|-i(G-R) \leq 2$ and $i(G-R) \geq 1$. Moreover, if $|R|=3$ and $i(G-R)=1$, then the two end-vertices of $e_{0}$ form an component of $G-R$ or two vertices of $R$ are joined by an edge of $G$.

If $G-e_{0}$ satisfies (3), then $G-e_{0}$ has the desired spanning tree by the inductive hypotheses. Hence we may assume that $G-e_{0}$ does not satisfy (3), which implies that 
there exists a subset $\emptyset \neq S \subset V(G)$ such that $i\left(G-e_{0}-S\right) \geq|S|$. Since $i\left(G-e_{0}-S\right) \leq$ $i(G-S)+2$ and (3), we have

$$
1 \leq|S|-i(G-S) \leq|S|-i\left(G-e_{0}-S\right)+2 \leq 2 .
$$

If $i(G-S) \geq 1$, then $R=S$ satisfies the first statement of the claim. If $i(G-S)=1$ and $|S|=3$, then $i\left(G-e_{0}-S\right)=3$ and $e_{0}$ joins two isolated vertices of $G-e_{0}-S$. Thus the two end-vertices of $e_{0}$ form an component of $G-S$, and hence $R=S$ also satisfies the second statement. Therefore we may assume $i(G-S)=0$.

If $|S|-i(G-S)=1$, then $|S|=1$ and $i\left(G-e_{0}-S\right)=1$ or 2 , and so $e_{0}$ joins the unique isolated vertex $y_{1}$ of $G-e_{0}-S$ to a non-isolated vertex $z_{1}$ of $G-e_{0}-S$, or $e_{0}$ joins the two isolated vertices $y_{1}$ and $y_{2}$ of $G-e_{0}-S$ (see Figure 2 (a)). Thus we have either $i\left(G-S \cup\left\{z_{1}\right\}\right)=\left|\left\{y_{1}\right\}\right|=1$ and $\left|S \cup\left\{z_{1}\right\}\right|-i\left(G-S \cup\left\{z_{1}\right\}\right)=1$ or $i\left(G-S \cup\left\{y_{2}\right\}\right)=\left|\left\{y_{1}\right\}\right|=1$ and $\left|S \cup\left\{y_{2}\right\}\right|-i\left(G-S \cup\left\{y_{2}\right\}\right)=1$. Therefore $R=S \cup\left\{z_{1}\right\}$ or $S \cup\left\{y_{2}\right\}$ satisfies the first tatement of the claim.

If $|S|-i(G-S)=2$, then $|S|=2, i\left(G-e_{0}-S\right)=2$ and $e_{0}$ joins the two isolated vertices $y_{3}$ and $y_{4}$ of $G-e_{0}-S$ (see Figure $\left.2(\mathrm{~b})\right)$. Thus $i\left(G-S \cup\left\{y_{4}\right\}\right)=\left|\left\{y_{3}\right\}\right|=1$ and $\left|S \cup\left\{y_{4}\right\}\right|-i\left(G-S \cup\left\{y_{4}\right\}\right)=3-1=2$. Hence $R=S \cup\left\{y_{4}\right\}$ satisfies the first statement of the claim. Furthermore, since $\operatorname{deg}_{G}\left(y_{4}\right) \geq 2$ and $y_{4}$ is an isolated vertex of $G-e_{0}-S$, there exists at least one edge joining $y_{4}$ to $S$. Hence $R=S \cup\left\{y_{4}\right\}$ also satisfies the second statement.
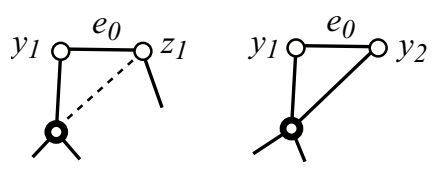

(a) $/ S /=1$
$S=\{\mathbf{0}$

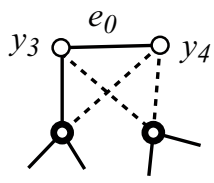

(b) $\mid S /=2$

Figure 2: $i(G-S)=0$; Broken lines are edges or not.

We define an integer $m$ by

$$
m=\min \{|X|-i(G-X) \mid R \subseteq X \subset V(G), i(G-X) \geq 1\},
$$

where $R$ is the vertex subset given in Claim 2. Since $i(G-X)<|X|$ by (3), we have $m \geq 1$. By Claim 2, we have $1 \leq m \leq 2$. Let $S_{0}$ be a maximal subset of $V(G)$ subject to $\left|S_{0}\right|-i\left(G-S_{0}\right)=m, R \subseteq S_{0}$ and $i\left(G-S_{0}\right) \geq 1$. Then we have

$$
m<|X|-i(G-X) \quad \text { for every } \quad S_{0} \subset X \subset V(G) \text { with } i(G-X) \geq 1 .
$$

Claim 3. Every non-trivial component $D$ of $G-S_{0}$ satisfies $i(D-T)<|T|$ for every subset $\emptyset \neq T \subseteq V(D)$. In particular, $|D| \geq 3$, and $D$ has a Hamiltonian cycle if $3 \leq|D| \leq 4$; and otherwise $D$ has a spanning tree with leaf distance at least four. 
Let $D$ be a non-trivial component of $G-S_{0}$. For every $\emptyset \neq T \subset V(D)$, by (5) we have

$$
m=\left|S_{0}\right|-i\left(G-S_{0}\right)<\left|S_{0} \cup T\right|-i\left(G-\left(S_{0} \cup T\right)\right),
$$

which implies $i(D-T)<|T|$. Hence the Claim follows by induction.

Before going to the next step, we need some definition. Let $T$ be a rooted tree with root $v$. Then for every vertex $x \in V(T)-v$, we define the parent of $x$ as the neighbor of $x$ lying on the $x-v$ path. Similarly, we define the children of $x$ as the neighbors of $x$ not lying on the $x-v$ path. Note that there exists exactly one parent for every $x \in V(T)-v$, but there may exist none or more than one children for some $x \in V(T)-v$.

We shall consider the following two cases accroding to $m$.

Case 1. $m=1$.

In this case, $\left|S_{0}\right|=i\left(G-S_{0}\right)+1$. Let $B$ be the bipartite graph with vertex set $I\left(G-S_{0}\right) \cup S_{0}$ and edge set $E_{G}\left(I\left(G-S_{0}\right), S_{0}\right)$, which is the set of edges of $G$ joining $I\left(G-S_{0}\right)$ to $S_{0}$. If $\left|N_{B}\left(X^{\prime}\right)\right| \leq\left|X^{\prime}\right|$ for some subset $\emptyset \neq X^{\prime} \subseteq I\left(G-S_{0}\right)$, then $i\left(G-N_{B}\left(X^{\prime}\right)\right) \geq$ $\left|X^{\prime}\right| \geq\left|N_{B}\left(X^{\prime}\right)\right|$, which contradicts (3). Hence

$$
\left|N_{B}(X)\right|>|X| \quad \text { for all } \emptyset \neq X \subseteq I\left(G-S_{0}\right) .
$$

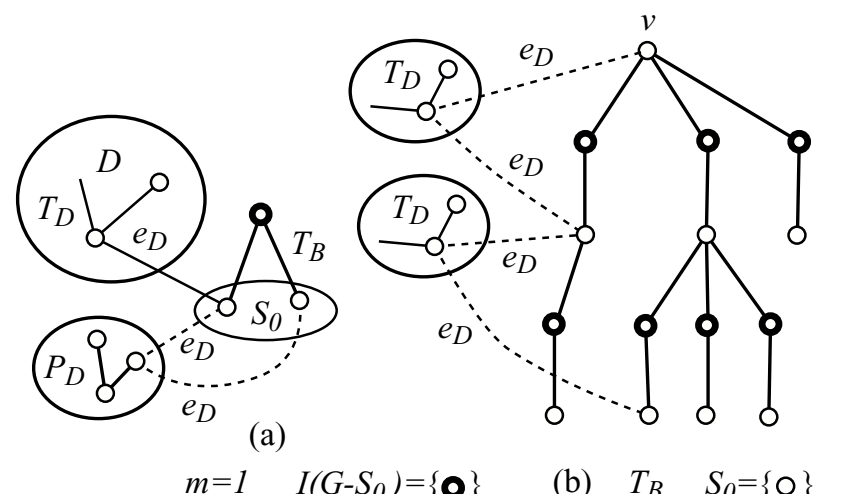

Figure 3: $S_{0}, I\left(G-S_{0}\right)$ and a spanning tree $T_{B}$.

By Lemma $4, B$ has a spanning tree $T_{B}$ such that all the leaves of $T_{B}$ are contained in $S_{0}$. If all the vertices in $S_{0}$ are leaves of $T_{B}$, then $\left|S_{0}\right|=2$, and there exists at least one non-trivial component of $G-S_{0}$ by Claim 1. Every non-trivial component $D$ of $G-S_{0}$ has a Hamiltonian cycle $C_{D}$ or a spanning tree $T_{D}$ with leaf distance at least four by Claim 3, and $G$ has an edge $e_{D}$ joining $D$ to a vertex of $S_{0}$ (Figure 3 (a)). If $D$ has a Hamiltonian cycle $C_{D}$, then $D$ has a Hamiltonian path $P_{D}$ such that $e_{D}$ joins a leaf of $P_{D}$ to $S_{0}$. Hence

$$
T=\bigcup_{D}\left(P_{D}+e_{D} \text { or } T_{D}+e_{D}\right)+T_{B}
$$


where the union is taken over all the non-trivial components $D$ of $G-S_{0}$, is the desired spanning tree of $G$ with leaf distance at least four (see Figure 3 (a)).

Therefore we may assume that $S_{0}$ contains a vertex $v$ that is not a leaf of $T_{B}$. We regard $T_{B}$ as a rooted tree with root $v$. Since all the leaves of $T_{B}$ are contained in $S_{0}$ and $m=\left|S_{0}\right|-i\left(G-S_{0}\right)=1$, for every vertex $y \in I\left(G-S_{0}\right)$, there exists exactly one child of $y$ in $S_{0}$ (Figure $2(\mathrm{~b})$ ). Let $D$ be a non-trivial component of $G-S_{0}$. By the same argument as above, $D$ has a Hamiltonian path $P_{D}$ or a spanning tree $T_{D}$ with leaf distance at least four, and a leaf of $P_{D}$ or a vertex of $T_{D}$ are joined to $S_{0}$ by an edge $e_{D}$ of $G$. Then

$$
T=\bigcup_{D}\left(P_{D}+e_{D} \text { or } T_{D}+e_{D}\right)+T_{B}
$$

where the union is taken over all the non-trivial components $D$ of $G-S_{0}$, is the desired spanning tree of $G$ with leaf distance at least four.

Case 2. $m=2$.

Let $B, T_{B}, D, P_{D}, T_{D}$ and $e_{D}$ be the same as in the proof of Case 1 . Note that the existence of them are guaranteed by the same argument as in the proof of Case 1.

If all the vertices in $S_{0}$ are leaves of $T_{B}$, then $\left|S_{0}\right|=3$ and $i\left(G-S_{0}\right)=1$. Put $S_{0}=\left\{u_{1}, u_{2}, u_{3}\right\}$ and $I\left(G-S_{0}\right)=\{w\}$ (see Figure 4 (a)). By Calim 2, $|R| \leq\left|S_{0}\right|=3$ and by the definition of $m$, we have $2=m \leq|R|-i(G-R) \leq 2,|R|=3$ and $i(G-R)=1$, which imply $S_{0}=R$. By the second statement of Claim 2 and Claim 3, two vertices of $S_{0}$ are joined by an edge of $G$. Without loss generality, we may assume that $u_{1}$ and $u_{2}$ are joined by an edge of $G$ (see Figure 4 (a)).

Assume that there exists a non-trivial component $D$ of $G-S_{0}$ such that the edge $e_{D}$ joins $D$ to $u_{3}$. If there exists another non-trivial $D$ of $G-S_{0}$ which is joined to $u_{i} \in\left\{u_{1}, u_{2}\right\}$ by an edge $e_{D}$, then for a spanning tree $T_{B}=\left\{u_{3} w, w u_{j}, u_{j} u_{i}\right\}$ of $B$, where $\left\{u_{i}, u_{j}\right\}=\left\{u_{1}, u_{2}\right\}$,

$$
T=\bigcup_{D}\left(P_{D}+e_{D} \text { or } T_{D}+e_{D}\right)+T_{B}
$$

forms the desired spanning tree of $G$. If for every non-trivial component $D$ of $G-S_{0}$, the edge $e_{D}$ joins $D$ to $\left\{u_{1}, u_{2}\right\}$, then by choosing $u_{i} \in\left\{u_{1}, u_{2}\right\}$ so that at least one $e_{D}$ is incident with $u_{i}$ and by letting $T_{B}=\left\{u_{3} w, w u_{j}, u_{j} u_{i}\right\}$, where $\left\{u_{i}, u_{j}\right\}=\left\{u_{1}, u_{2}\right\}$, $T=\bigcup_{D}\left(P_{D}+e_{D}\right.$ or $\left.T_{D}+e_{D}\right)+T_{B}$, forms the desired spanning tree of $G$. Therefore we may assume that $S_{0}$ contains at least one vertex of $T_{B}$ which is not a leaf of $T_{B}$.

Let $v \in S_{0}$ be a vertex that is not a leaf of $T_{B}$. We regard $T_{B}$ as a rooted tree with root $v$. Since all the end-vertices of $T_{B}$ are contained in $S_{0}$ and $m=\left|S_{0}\right|-\left|I\left(G-S_{0}\right)\right|=2$, $I\left(G-S_{0}\right)$ has exactly one vertex $x$ that has exactly two children, say $y_{1}$ and $y_{2}$, and every other vertex of $I\left(G-S_{0}\right)-x$ has exactly one child. (Fig. 2 (b) (c)). If at least one of $\left\{y_{1}, y_{2}\right\}$ is not an pendant vertex of $T_{B}$ (Fig. $2(\mathrm{c})$ ), then we can obtain the desired spanning tree $T=\bigcup_{D}\left(P_{D}+e_{D}\right.$ or $\left.T_{D}+e_{D}\right)+T_{B}$, which is given in the proof of Case 1 . Hence we hereafter assume that both $y_{1}$ and $y_{2}$ are end-vertices of $T_{B}$ (Figure 2 (b)).

If $G$ has an edge joining $\left\{y_{1}, y_{2}\right\}$ to a non-trivial component $D$ of $G-S_{0}$, then by choosing $e_{D}$ to be such an edge, we obtain the desired spanning tree $T=\bigcup_{D}\left(P_{D}+\right.$ 


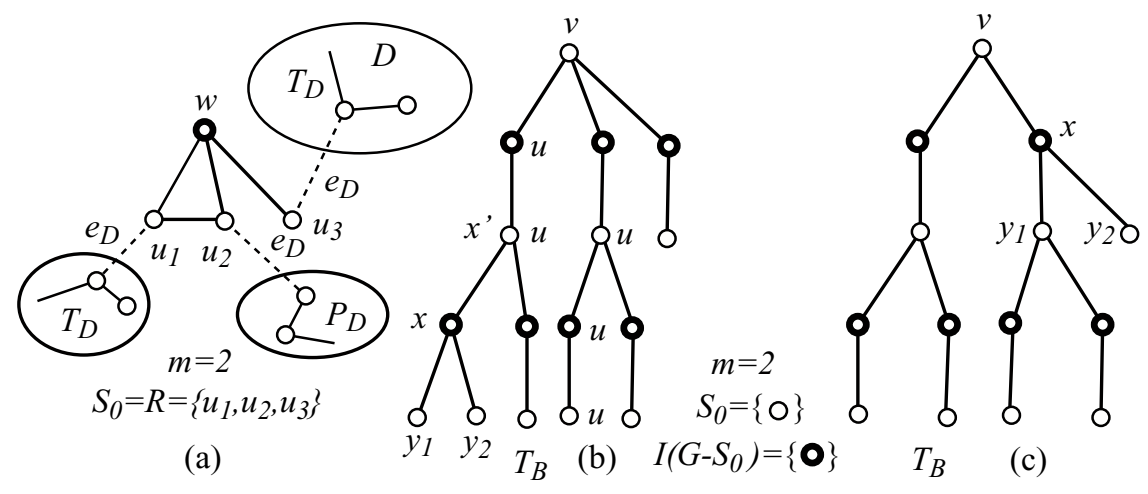

Figure 4: $S_{0}, I\left(G-S_{0}\right)$ and a spanning tree $T_{B}$.

$e_{D}$ or $\left.T_{D}+e_{D}\right)+T_{B}$ of $G$. So we may assume that no edge of $G$ joins $\left\{y_{1}, y_{2}\right\}$ to $V(G)-\left(S_{0} \cup I\left(G-S_{0}\right)\right)$. If $y_{1}$ and $y_{2}$ are adjacent in $G$, then

$$
T=\bigcup_{D}\left(P_{D}+e_{D} \text { or } T_{D}+e_{D}\right)+T_{B}+y_{1} y_{2}-x y_{2}
$$

is the desired spanning tree of $G$. Thus we may assume that $y_{1}$ and $y_{2}$ are not adjacent in $G$. Since $\operatorname{deg}_{G}\left(y_{1}\right) \geq 2, y_{1}$ is adjacent to a vertex $u$ of $T_{B}$, which is not $y_{2}$. Let $x^{\prime}$ be the parent of $x$. Then

$$
T=\bigcup_{D}\left(P_{D}+e_{D} \text { or } T_{D}+e_{D}\right)+T_{B}+y_{1} u-x x^{\prime}
$$

is the desired spanning tree of $G$.

Consequently the proof is complete.

\section{References}

[1] A. Kaneko, Spanning trees with constraints on the leaf degree, Discrete Applied Math., 115 (2001), 73-76.

[2] A. Kaneko and K. Yoshimoto, On spanning trees with restricted degrees. Inform. Process. Lett. 73 (2000),163-165.

[3] M.N. Ellingham, Y. Nam, H.-J. Voss, Connected $[g, f]$-factors, J. Graph Theory, 39 (2002), 62-75.

[4] M. Kouider and P. D. Vestergaard, Connected factors in graphs - a survey, Graphs and Combi., 21 (2005) 1-26.

[5] S. Win, On a connection between the existence of $k$-trees and the toughness of a graph, Graphs and Combin., 5 (1989) 201-205. 